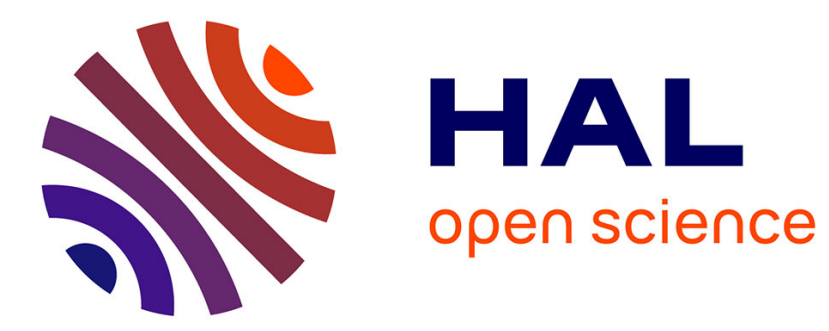

\title{
Smart management of technologies: predictive maintenance of industrial equipment using wireless sensor networks
}

Andrey I. Vlasov, Pavel V. Grigoriev, Aleksey I. Krivoshein, Vadim A. Shakhnov, Sergey S. Filin, Vladimir S. Migalin

\section{To cite this version:}

Andrey I. Vlasov, Pavel V. Grigoriev, Aleksey I. Krivoshein, Vadim A. Shakhnov, Sergey S. Filin, et al.. Smart management of technologies: predictive maintenance of industrial equipment using wireless sensor networks. Entrepreneurship and Sustainability Issues, 2018, 6 (2), pp.489-502. 10.9770/jesi.2018.6.2(2) . hal-02342832

\section{HAL Id: hal-02342832 \\ https://hal.science/hal-02342832}

Submitted on 1 Nov 2019

HAL is a multi-disciplinary open access archive for the deposit and dissemination of scientific research documents, whether they are published or not. The documents may come from teaching and research institutions in France or abroad, or from public or private research centers.
L'archive ouverte pluridisciplinaire HAL, est destinée au dépôt et à la diffusion de documents scientifiques de niveau recherche, publiés ou non, émanant des établissements d'enseignement et de recherche français ou étrangers, des laboratoires publics ou privés. 


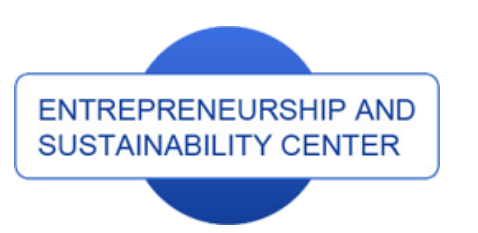

Publisher

http://jssidoi.org/esc/home

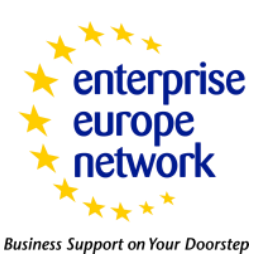

CASPA

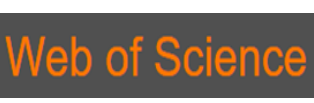

\title{
SMART MANAGEMENT OF TECHNOLOGIES: PREDICTIVE MAINTENANCE OF INDUSTRIAL EQUIPMENT USING WIRELESS SENSOR NETWORKS*
}

\author{
Andrey I. Vlasov ${ }^{1^{*}}$, Pavel V. Grigoriev ${ }^{2}$, Aleksey I. Krivoshein ${ }^{3}$, Vadim A. Shakhnov ${ }^{4}$, \\ Sergey S. Filin ${ }^{5}$, Vladimir S. Migalin ${ }^{6}$ \\ 1,3,4 Bauman Moscow State Technical University \\ 5, Vtoraya Baumanskaj Str., Moscow, 105005, Russian Federation \\ 1.2,3,5,6 LLC “Konnekt”, \\ 2/15, Lasenki Str., Moscow, 119619, Russian Federation \\ E-mails: ${ }^{1}$ vlasov@iu.4.ru; ${ }^{4}$ shakhnov.@bmstu.ru, ${ }^{2-6}$ info@iu.4.ru
}

Received 15 August 2018; accepted 20 October 2018; published 30 December 2018

\begin{abstract}
One of the most important problems of creating new and also modernizing and operating the existing industrial equipment is to provide it with technical diagnostic tools. In modern systems, most diagnostic problems are solved by vibration monitoring methods, and they form the basis of this process. For several years already, when creating new responsible equipment, many manufacturers have completed it with monitoring and diagnostic systems, often integrating them functionally with automatic control systems. This paper discusses the methods of servicing industrial equipment, focusing on predictive maintenance, also known as actual maintenance (maintenance according to the actual technical condition). The rationale for the use of wireless systems for data collection and processing is presented. The principles of constructing wireless sensor networks and the data transmission protocols used to collect statistical information on the state of the elements of industrial equipment, depending on the field of application, are analyzed. The purpose of the study is to substantiate the feasibility of using wireless sensor networks as technical diagnostic tools from both economic and technical points of view. The result is the proposed concept of the predictive maintenance system. The paper substantiates the model of optimization of predic-tive repair using wireless sensor networks. This approach is based on minimizing the costs of maintenance of equipment. The presented concept of a system of predictive maintenance on the basis of sensor networks allows real-time analysis of the state of equipment. The approach allows implementing smart management of technologies in companies for ensuring stability of functioning.
\end{abstract}

Keywords: management of technologies; monitoring of technological processes; industrial equipment; predictive repair; smart management concept.

Reference to this paper should be made as follows: Vlasov, A.I.; Grigoriev, P.V.; Krivoshein, A.I; Shakhnov, V.A.; Sergey S. Filin, S.S.; Migalin, V.S. 2019. Smart management of technologies: predictive maintenance of industrial equipment using wireless sensor networks, Entrepreneurship and Sustainability Issues 6(2): 489-502. http://doi.org/10.9770/jesi.2018.6.2(2)

JEL Classifications: D24; C14; C80

\footnotetext{
* The research was conducted with the support of the Ministry of Education and Science of Russia within the framework of the project under the Agreement No.14.579.21.0142 UID RFMEFI57917X0142.
} 


\section{Introduction}

Modern conditions imply a new development model based on human capital, innovation and high technologies. Flexibility and speed of innovation are the key success factors. These trends are typical of both social life and industrial production processes. In this context, smart environments perform a special role: in fact, they serve as a frame which will be added some infrastructure that can guarantee new product quality. Smart production involves using machines capable of exchanging information with other production systems and operating with a high degree of autonomy; using the industrial Internet of Things; and utilizing Big Data cloud service and technology. However, the most important link in this chain is integration platforms of an enterprise which are aimed at receiving data from equipment, analyzing and aggregating them. One of the sides of this issue is the system of industrial equipment that requires its condition to be constantly monitored. At present, several methods of servicing equipment have been formed, among which three basic ones can be distinguished: emergency, routine and predictive. The first kind is the maintenance of equipment after its failure Brüel \& Kjær (1991). This approach is justified in the maintenance of simple, cheap equipment, when reservation is available, and replacement will be cheaper than repair work to restore equipment.

The second type of maintenance is planned preventive maintenance of equipment in accordance with the regulations (Brüel \& Kjær, 1991). In this case, the maintenance is carried out in accordance with the manufacturer's recommendations at regular intervals. The equipment maintenance work is performed with a certain period, which is determined by statistical analysis and, in accordance with the regulatory documents, the period typically equals the time during which at least $98 \%$ of the equipment operates without failure (Brüel \& Kjær, 1991).

When servicing according to the regulations, it would seem that the opportunity to use the guarantee of the manufacturer is not lost, at least. However, it should be taken into account that almost $50 \%$ of all technical maintenance works according to the regulations are carried out without their factual need. In addition, for many types of equipment, maintenance and repair according to the regulations do not reduce the frequency of their failure. Moreover, the reliability of the equipment operation after maintenance, if the latter involves disassembling the mechanism or replacing parts, is often reduced, sometimes temporarily, until the moment of their running-in, and sometimes this decrease in reliability is caused by the manifestation of installation defects which were missing before the maintenance.

The third type of maintenance, which is discussed in detail in the present paper, is predictive maintenance, also known as maintenance according to the actual technical state (Rawi, 2010; Stone, 2007; Vlasov et al, 2017; Mobley, 2002). With this type of service, the state of the equipment is monitored continuously or periodically. Depending on the results obtained, a forecast is made of the technical condition of the equipment and maintenance programs are formed. The predictive maintenance systems can predict the state of the system based on the current state of equipment and determine the necessary maintenance activities. Thus, the probability of an unscheduled system failure is minimized.

That is why in the present research the main attention is paid to the analysis of the model for evaluating the effectiveness of predictive services using wireless sensor networks. Presently, the problem of constructing distributed systems for data collection and monitoring of technological processes and individual equipment is more urgent than ever. This leads to the need to develop methods for constructing intelligent monitoring of technical industrial systems. 


\section{Literature review}

In connection with monitoring industrial systems in order to prevent malfunctions, the authors will analyze a model of a predictive maintenance system based on a wireless sensor network (Vlasov et al, 2017; Sharapov \& Polischuk, 2012; Kozlova, 2009; Bogdanov \& Basov, 2012; Shakhnov et al, 2013). Sensors are used as the elements of systems recording various data. Sensors are means of measurement designed to generate signals of measurement information in a form that is convenient for transmission, further transformation, processing and/or storage, but which cannot be directly perceived by the observer (Kozlova, 2009). Depending on the signal transmission medium, the sensors can be wired and wireless. The use of wired systems is not always effective due to a high cost of installation and commissioning, as well as technical maintenance. In addition, in some situations the installation of wired sensors is altogether impossible for technological or organizational reasons. Among the advantages of wireless sensors there are minimal restrictions on their placement, the possibility of introducing and modifying the network of such sensors on the operated object without interfering with the operation process, reliability and fault tolerance of the entire system in the event of disruption of individual connections between nodes (Bogdanov \& Basov, 2012).

The predictive maintenance systems based on the wireless sensor network, in contrast to other types of technical maintenance, save the data obtained in the monitoring process, which allows usingadvanced methods of monitoring the technical state and analyzing the data in real time, as well as making forecasts for the technical condition of the equipment.

For a long time, systems with wired communication channels provided a reliable transmission medium and a high speed with a long service life. Despite many advantages, wired solutions also have a number of limitations, which gradually make them less attractive compared to wireless technologies. Among these limitations, one can identify the following:

- spatial arrangement of sensors;

- the costs of maintaining communication channels;

- the deployment time of wired networks.

These three major shortcomings of wired transmission explain the fact that wireless networks, radio networks are very rapidly gaining momentum. This is explained by the convenience of their use, low cost and acceptable transmission capacity.

When building a wireless sensor network, one needs to choose a data transfer protocol,since, depending on the field of application of the wireless sensor network, one or another protocol or standard can be used to interact with wireless sensors of the reading equipment (Vlasov, Ivanov \& Kosolapov, 2011). Table 1 shows a comparison of the RF interface types for wireless tags/sensors. 
Table 1. Comparison of RF interface types for wireless tags / sensors

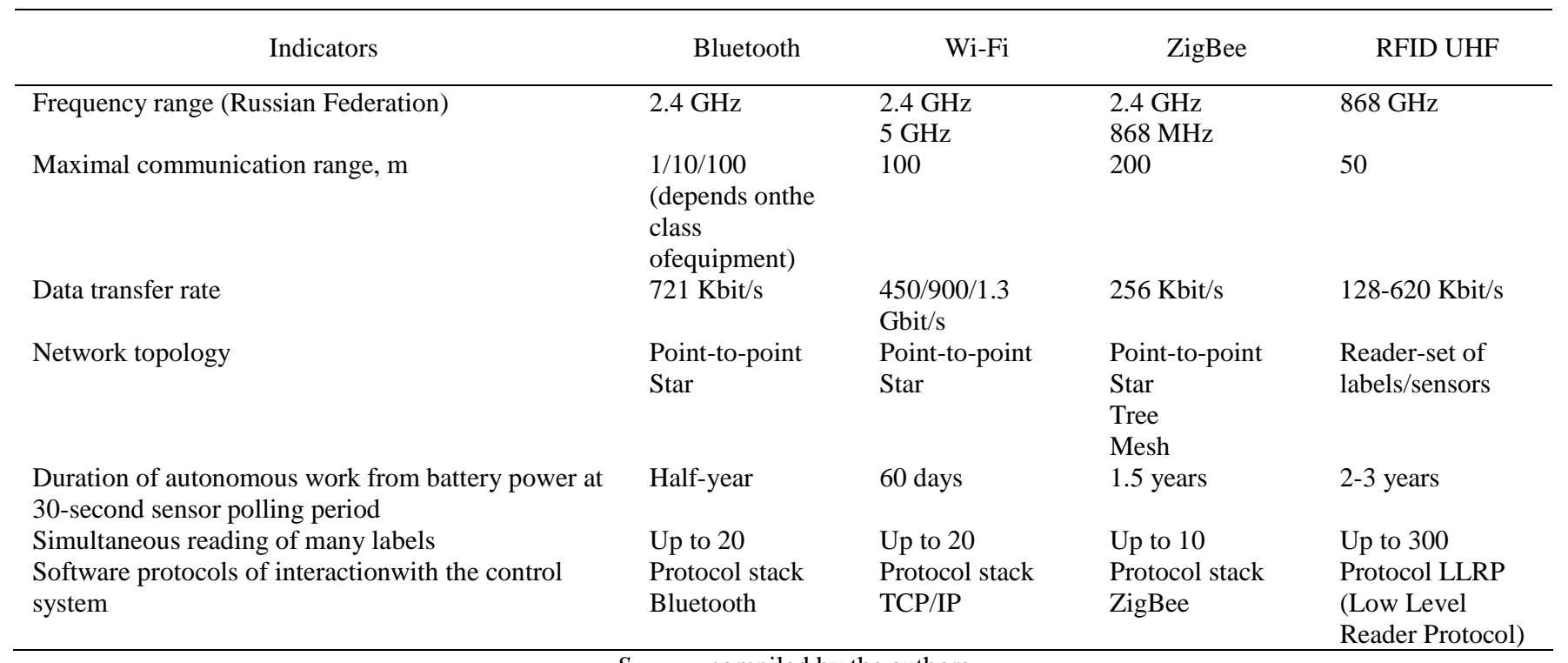

Source: compiled by the authors

A wireless sensor network consists of a set of spatially-distributed intelligent sensors designed to monitor physical parameters such as vibration, temperature, strain, pressure, etc. Each sensor node in the network performs the function of reading, processing and wireless transmission of data. The use of microelectromechanical system technology (MEMS) provides an opportunity for low-cost production of low-power multifunctional sensors of small size and low weight. The use of wireless sensors with autonomous power supply necessitates a detailed consideration of the principles of their construction, functional filling and used circuit solutions in accordance with the generalized structural scheme in Figure 1.

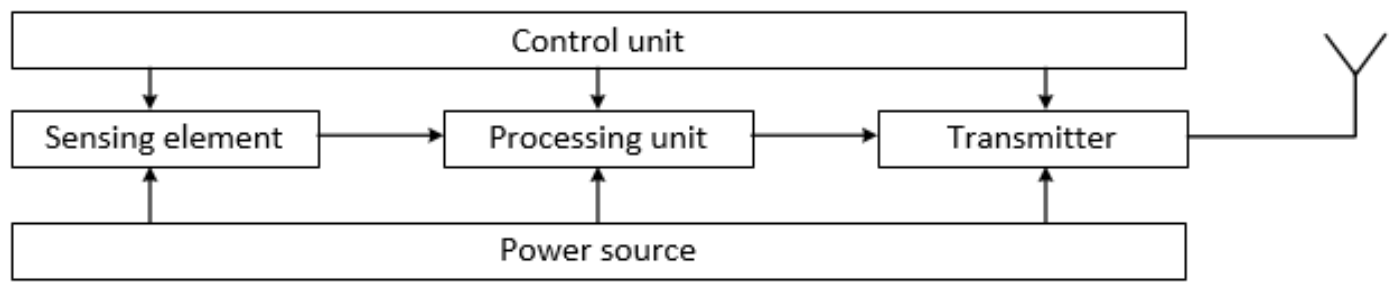

Fig. 1. Generalized block diagram of the wireless sensor Source: compiled by the authors

The registration of the monitored characteristics is carried out by the sensing element (SE) of the sensor. At the same time, to measure one and the same quantity, the sensors can be applied that use various physical processes of its transformation into electrical signals and various principles of action (Andreev, Vlasov \& Shakhnov, 2016; Freyden, 2005; Gotra \& Tchaikovsky, 1995; Yudin et al, 2017; Burduk \& Chlebus, 2009). The processing unit is designed to amplify an electrical signal and convert it to a form convenient for transmission. The functional filling of the processing units depends on the type of output signal. It should be noted that the criteria for achieving a minimum current consumption are contrary to the criteria for achieving maximum performance; therefore, micropower op amps have very modest speed parameters (Freyden, 2005; Gotra \& Tchaikovsky, 1995). 
According to the data presented in Table 1, it is proposed to use wireless sensor networks based on radio frequency identification (RFID) (Vlasov, Grigoriev \& Zhalnin, 2017b), since the off-line operation time and the number of simultaneously read labels are the main parameters when choosing a radio interface in the systems of predictive equipment maintenance.

\section{Methods}

\subsection{The concept of a predictive maintenance system based on sensor networks with an intelligent controller}

Intelligent controllers are increasingly used in distributed industrial systems of different purposes. Recently, to obtain data on the status of equipment, wireless sensor networks, which consist of various sensors including infrared sensors, acoustic sensors, vibration, shock and acceleration sensors, have been increasingly used (Vlasov, Grigoriev \& Zhalnin, 2017b; Freyden, 2005; Gotra \& Tchaikovsky, 1995; Yudin et al, 2017; Burduk \& Chlebus, 2009). Figure 2 presents a generalized conceptual scheme for obtaining data and analyzing the state of equipment in real time. In the process of work, continuous monitoring of the status of the modules takes place, as well as comparison of the obtained data from the sensors with readings during normal operation of the equipment, to determine possible malfunctions.

Figure 2 shows a generalized example of a monitoring object (1), on the equipment of which wireless sensor tags are installed. Abandoning wired sensors allows placing sensors in hard-to-reach places, thereby increasing the number of sensors themselves and the accuracy of the parameters obtained during the monitoring process. Sensor data are read and transmitted over the wireless network to external servers, where data are converted, stored and processed (2).

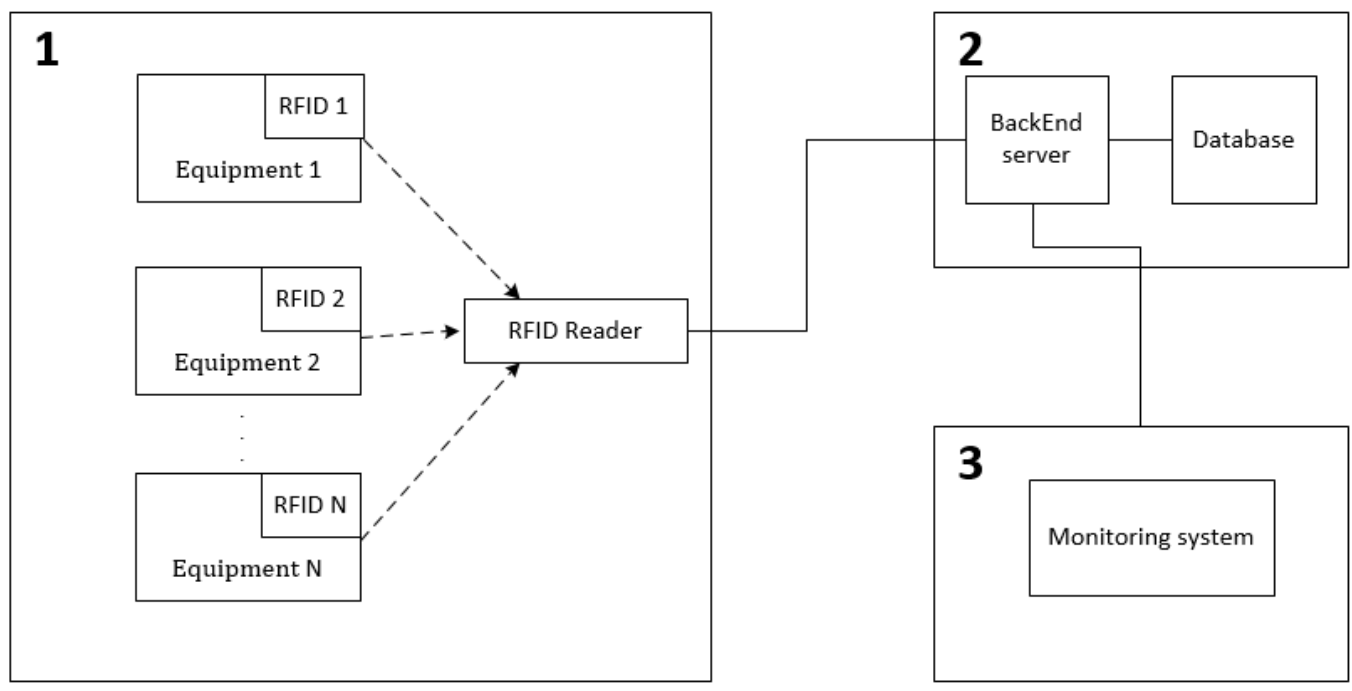

Fig. 2. Analysis of the state of equipment in the real time mode Source: compiled by the authors.

All data received from sensors is stored in a database located in the cloud. The data is required to present statistics. There is also a defect database, which includes information related to the defects of various devices, the correspondence of arising defects and the operating time on which such defects usually occur. Data obtained as a 
result of monitoring is processed and then sorted in the order of frequency of occurrence. This approach allows forming a program of predictive maintenance of equipment, as well as predicting the failure of any of its parts.

Saved data can be obtained at any time in the form of graphs or individual events and displayed on the screen of a computer, tablet or mobile phone (3).

Broad implementation of solutions for the implementation of the concept of "Internet of things" imposes a number of requirements on the approaches to the realization of the functions of predictive maintenance. To achieve this goal, intelligent controllers and multi-agent technologies are needed (Vlasov et al, 2017). To each object in the real world, a software agentis put into correspondence: an object with some degree of intelligence. Recently, systems have been developed based on KNX and HDL wired buses or Zwave and ZigBee wireless standards. Such solutions often have unused options and, at the same time, do not have priority important functions. The software and hardware complex should interact with the following subsystems: power supply, universal chassis, localization module. In general, the intelligent controller must provide:

- monitoring of engineering systems (collection of information with the construction of graphs and temporary reports on current data);

- processing of the obtained information (calculation of maintenance, predictive repair and replacement of elements);

- timely notification and assistance in decision-making;

- remote control of engineering systems in the presence of such functionality;

- $\quad$ self-diagnostics of the main nodes, monitoring results should be transferred to a single cloud storage.

Within the framework of the work, the concept of a universal intelligent communication platform for integrated embedded solutions is proposed, which allows connecting the decision module and heterogeneous sensor elements, localization and positioning systems into a single whole. In the "Internet of Things" paradigm, a logical level is added to the hardware subsystems, which allows transferring the computational tasks directly to the execution units themselves, and transmittingonly the processed data to the data collection and storage station. This task can be solved with the help of universal controllers. Such controllers have sufficient computing power to perform tasks not only of processing data received from field devices, but also tasks that require the implementation of complex calculation algorithms. Universal controllers can function as a buffer memory device: all measured and calculated values are put into the controller memory and stored for the required period. Thus, even if communication with the data collection station is lost, they will be buffered, and then transferred to the server when communication is resumed.

When solving practical problems, special attention is paid to ensuring that intelligent controllers are scalable and expandable to localize solutions for specific parameters. One can identify the following tasks, which should be addressed at the hardware level (controller level):

- transfer of signals from the field level devices (sensors) to engineering units and transfer of information to a data collection station;

- execution of algorithms for calculating physical quantities to take into account the obtained data;

- control of technical systems, taking into account the built-in algorithms, as well as according to the instruction of the head station;

- formation and transmission of warning and alarm signals.

The tasks of the data collection station (processing module) can be described in more detail as follows: structuring and formalization of measurement information, accumulation of the dynamics of changes; processing and analysis of incoming data; analysis of the obtained results of processing to identify non-typical behavior and search based 
on the results of the analysis of a list of possible causes and factors that may lead to the development of contingencies. The intelligent controllers implemented in this concept provide the basic functionality of predictive repair and can be integrated into the complexes implemented within the concept of the "Internet of Things"(IoT).

\subsection{A model for estimating the effectiveness of predictive repair}

Based on the analysis of the solutions presented in the fundamental works on predictive repair (Vlasov et al, 2017a; Burduk \& Chlebus, 2009; Vlasov, Grigoriev \& Zhalnin, 2017b; Curcuru et al, 2017; Rausand \& Hoyland, 2004; Lu \& Meeker, 1993; Crowder \& Lawless, 2007; Baptista et al, 2018), to assess the effectiveness of predictive repair, it is proposed to use the model based on minimizing the costs of maintenance, diagnostics and taking into account the risks of failure of individual components:

$$
c\left(S_{p}\right)=\frac{C_{p r}+L_{f} \cdot F\left(S_{p}\right)}{\bar{t}\left(S_{p}\right)}+c_{d}
$$

where $C_{p r}$ is the cost of predictive maintenance; $S_{p}$ is the value of the signal received from the sensors in the process of monitoring the state of the equipment (is a random quantity); $F\left(S_{p}\right)$ is the distribution function of the probability of failure in relation to the signal from the sensor; ${ }^{L_{f}}$ are losses due to the risks of failure; ${ }^{c_{d}}$ are unit costs for monitoring the condition of equipment to obtain a diagnostic signal; $\bar{t}\left(S_{p}\right)$ is the average operating time of the equipment before failure, which is determined by the formula (2):

$$
\bar{t}\left(S_{p}\right)=\frac{1}{n}\left[\sum_{i=1}^{m\left(S_{p}\right)} t_{i}\left(S_{p}\right)+\sum_{j=1}^{n-m\left(S_{p}\right)} t_{j}\left(S_{p}\right)\right] \text {, }
$$

where $t_{i}\left(S_{p}\right)$ is the time of operation of the $i$-th object with the normal value $S_{p}$ of the signal; $t_{j}\left(S_{p}\right)$ is the operating time of the $j$-th object, which failed before the diagnostic signal from the sensor reached the state $S_{p}$; $m\left(S_{p}\right)$ is the number of objects that reach the state $S_{p}$ without failures; $n$ is the total number of sensors for monitoring.

To obtain the average time until the equipment fails, it is necessary to conduct tests that include diagnostic measurements. When analyzing the obtained data, it is necessary to calculate the average time from the moment the signal about the unstable operation of the equipment is received until it completely fails. In this case, applying the equation (2), it is possible to calculate the average operating time of the equipment from the moment of receiving the signal about unstable operation until it completely fails.

When calculating the average operating time, it is proposed to apply a simplified model in which the deterioration of the technical state (change of the diagnostic signal) runs along a straight line from the initial state $S_{p z}$ to the limit value of the technical state (to failure) $S_{p f i}$ of the $i$-th object. The calculation of $t_{i}\left(S_{p}\right)$ is carried out according to the formula:

$$
t_{i}\left(S_{p}\right)=t_{i}\left(S_{p f i}\right) \frac{S_{p i}-S_{z}}{S_{p f i}-S_{z}}
$$


Figure 3 shows a graphical method for determining the input data for calculating the average operating time of the equipment, depending on the diagnostic signals from the sensors in the case of predictive maintenance.

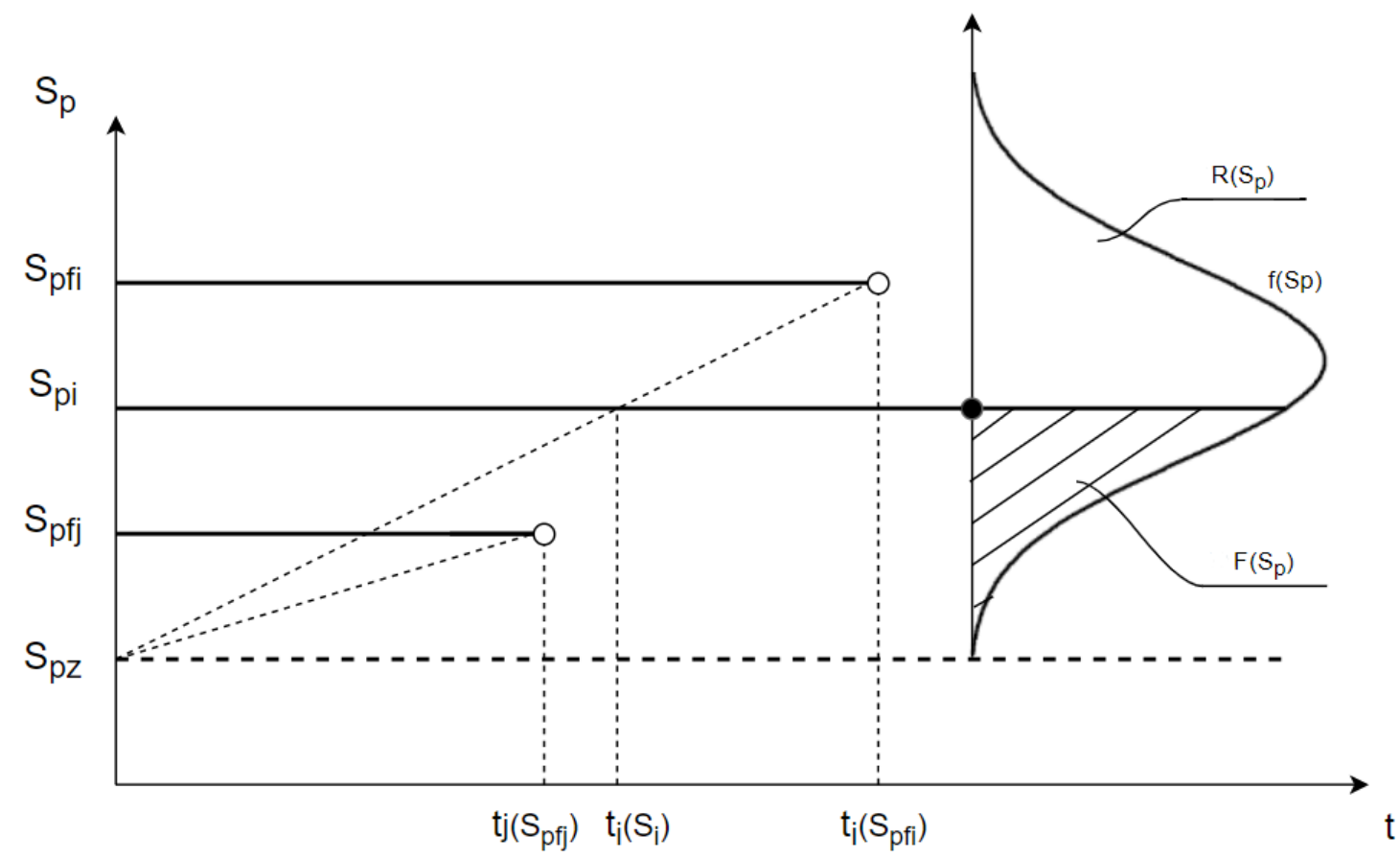

Fig. 3. Graphical method for determining the input data for calculating the average operating time of the equipment, depending on the diagnostic signals from the sensors Source: compiled by the authors.

As the unit costs for monitoring the state of the equipment for obtaining a diagnostic signal, one can consider the costs of deploying the wireless sensor network and the service; this can be described according to the formula (4):

$$
c_{d}=l \cdot\left(C_{t}+C_{s}\right)+k \cdot C_{r} .
$$

Thus, the model for optimizing predictive repair using wireless sensor networks, based on minimizing the costs of equipment maintenance, can be described according to the formula (5):

$$
\begin{aligned}
& c\left(S_{p}\right)=\frac{C_{p r}+L_{f} \cdot F\left(S_{p}\right)}{\bar{t}\left(S_{p}\right)}+c_{d}=\frac{C_{p r}+L_{f} \cdot F\left(S_{p}\right)}{\frac{1}{n}\left[\sum_{i=1}^{m\left(S_{p}\right)} t_{i}\left(S_{p}\right)+\sum_{j=1}^{n-m\left(S_{p}\right)} t_{j}\left(S_{p}\right)\right]}+c_{d}= \\
& =\frac{C_{p r}+L_{f} \cdot F\left(S_{p}\right)}{\frac{1}{n}\left[\sum_{i=1}^{m\left(S_{p}\right)} t_{i}\left(S_{p}\right)+\sum_{j=1}^{n-m\left(S_{p}\right)} t_{j}\left(S_{p}\right)\right]}+l \cdot\left(C_{t}+C_{s}\right)+k \cdot C_{r}
\end{aligned}
$$

where $C_{p r}$ is the cost of predictive maintenance; $S_{p}$ is the value of the signal received from the sensors in the process of monitoring the state of the equipment (is a random quantity); $F\left(S_{p}\right)$ is the distribution function of the 
probability of failure in relation to the signal from the sensor; ${ }^{L_{f}}$ are losses due to the risks of failure; ${ }^{c_{d}}$ are unit costs for monitoring the condition of equipment to obtain a diagnostic signal; ${ }^{-}\left(S_{p}\right)$ is the average time of operation of the equipment before failure, which is determined by the formula (2); $l$ is the number of sensor tags with sensors; $C_{t}$ is the cost of one sensor tag; $C_{s}$ is the cost of one sensor; $k$ is the number of readers; $C_{r}$ is the cost of purchasing and installing a single reader.

\section{Results and Discussion}

The proposed module of intelligent monitoring of technical systems was tested in the framework of monitoring the state of electric motors. If the motor is in operation for a long time, various defects may appear in it. If they are not remedied in time, the electric motor will fail. In any electric motor there are rolling bearings or friction bearings. The estimated life of rolling bearings on average does not exceed 8,000-10,000 h, which is slightly more than one year of continuous operation. In practice, rolling bearings often serve more than this period. Analyzing the signals from the vibration sensors installed on the supporting bearing of electric machines, defects in their condition were detected. When choosing defects that are described in this section, the authors proceeded from the following definition: if a defect can be diagnosed by installing vibration sensors on the supporting bearings, then its description is included in this section. Figure 4 shows a histogram of the appearance of defects, depending on the running time of the electric motor.

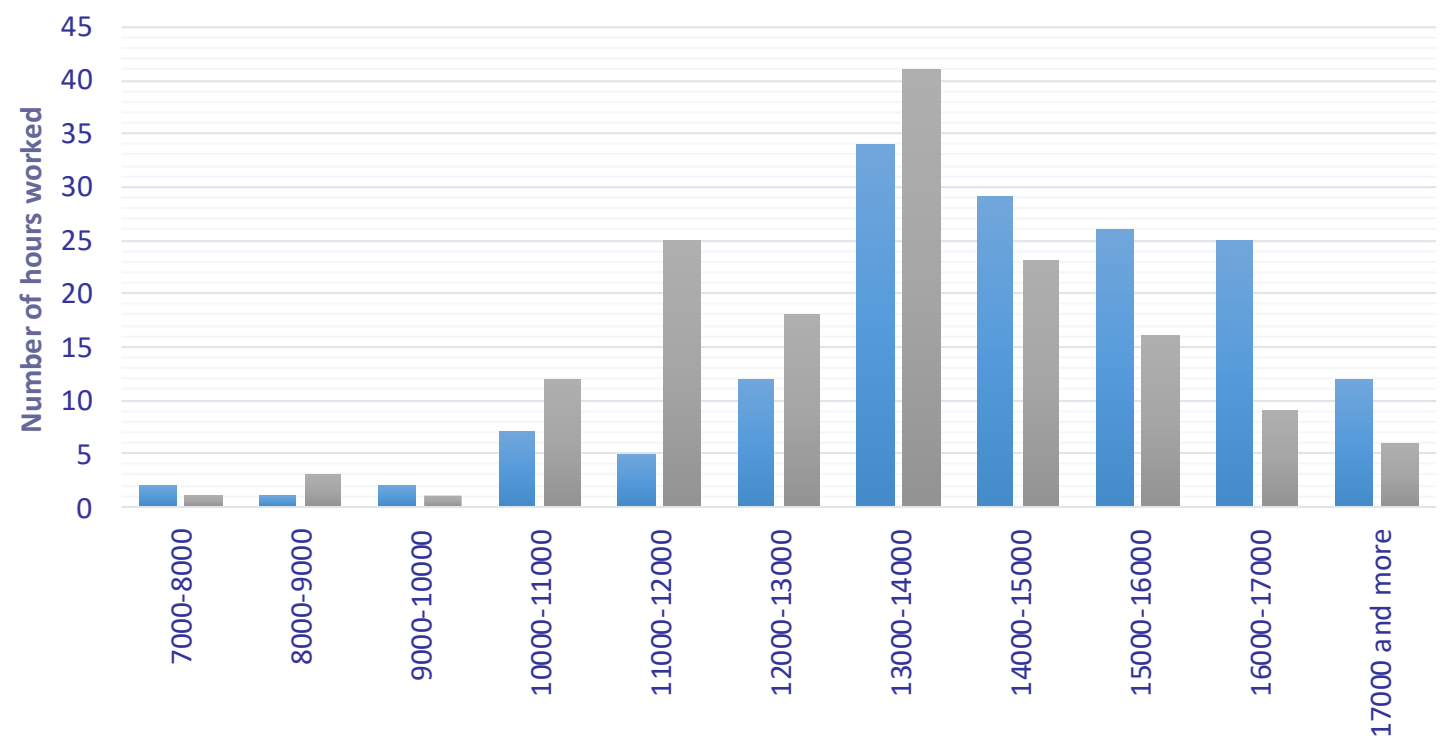

Number of defects detected

Number of failed motor bearings Number of received signals from sensors

Fig 4. The number of defects that occur depending on the running time of the electric motor Source: compiled by the authors.

During monitoring, the readings were taken from AC motors. Out of 155 cases of defects, only 3 cases occurred before the operator received a signal about a possible failure of the equipment. As can be seen from the graph 
depicted in Figure 5, real-time monitoring allows preventing up to $97 \%$ of the cases of failure of this type of equipment.

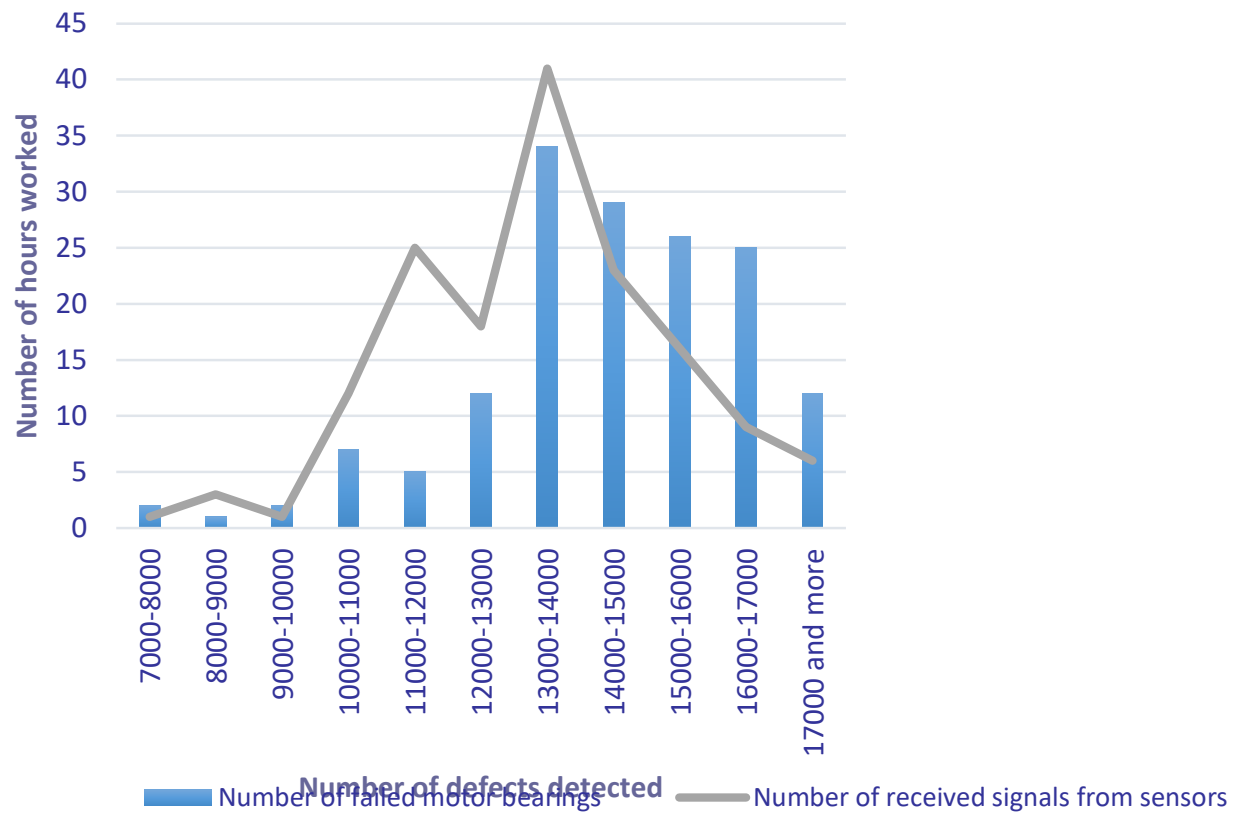

Fig. 5. The number of defects that occur depending on the running time of the electric motor Source: compiled by the authors.

Thus, analyzing the obtained data, it is possible to plot the graph as shown in Figure 6 and graphically calculate the average time of operation of the equipment, depending on the diagnostic signals from the sensors in the case of predictive service. 
The International Journal

ENTREPRENEURSHIP AND SUSTAINABILITY ISSUES

ISSN 2345-0282 (online) http://jssidoi.org/jesi/

2018 Volume 6 Number 2 (December)

http://doi.org/10.9770/jesi.2018.6.2(2)

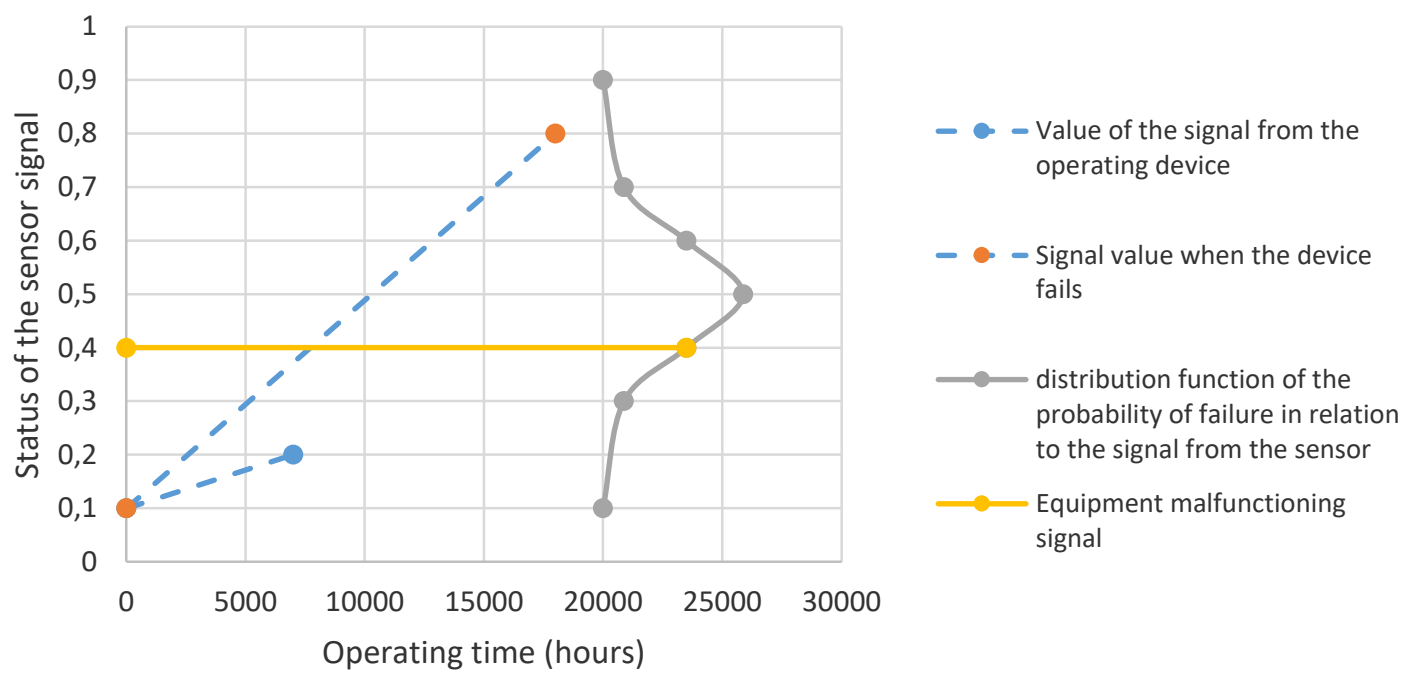

Fig. 6. Graphical method for determining the input data for calculating the average operating time of the equipment, depending on the diagnostic signals from the sensors Source: compiled by the authors.

The presented concept of a system of predictive maintenance on the basis of sensor networks allows real-time analysis of the state of equipment. According to the data received from sensors, a program of maintenance of equipment is formed. In the future, this approach is supposed to be developed taking into account the microminiaturization of the elements of the intellectual controller, the creation of a specialized element base that allows diagnostics of individual subsystems in real time with intelligent processing functions (Whitaker et al, 2018; Amruthnath \& Gupta, 2018).

\section{Conclusion}

Enhancing the efficiency of industry and infrastructure through the use of smart electronic systems - the so-called smart environments - is one of the most well-known avenues for technological development. The distinctive features of smart environments (Cook \& Das, 2005) are remote control, communication between devices, sophisticated device functionality, developed network standards, obtaining information through smart sensory networks and its distribution, predictive and decision-making abilities. Currently, manufacturing companies gaining experiences in production in networks and smart logistics and develop new organisational structures and business models which better benefit from the new technologies and which adapt faster to the rapidly changing network environments (Prause \& Atari, 2017).

Predictive maintenance of equipment is aimed at predicting the place and time of probable breakdown, as well as avoiding downtime and reducing maintenance costs. The use of wireless communication channels in the monitoring system makes it possible to deploy the sensor network in the shortest possible time, regardless of the spatial arrangement of the sensors. A model for optimizing predictive maintenance of equipment using wireless sensor networks based on minimizing the costs of maintenance, diagnostics and deployment of the equipment monitoring system is proposed. The presented concept of a system of predictive maintenance on the basis of sensor networks allows real-time analysis of the state of equipment. 
The International Journal

ENTREPRENEURSHIP AND SUSTAINABILITY ISSUES

ISSN 2345-0282 (online) http://jssidoi.org/jesi/

2018 Volume 6 Number 2 (December)

http://doi.org/10.9770/jesi.2018.6.2(2)

\section{Acknowledgments}

The research was conducted with the support of the Ministry of Education and Science of Russia within the framework of the project under the Agreement No.14.579.21.0142 UID RFMEFI57917X0142.

\section{References}

Amruthnath, N.; \& Gupta, T. 2018. A research study on unsupervised machine learning algorithms for early fault detection in predictive maintenance, In: 2018 5th International Conference on Industrial Engineering and Applications, IEEE, 355-361, http://dx.doi.org/10.13140/RG.2.2.28822.24648.

Andreev, K.A.: Vlasov, A.I.; \& Shakhnov, V.A. 2016. Silicon pressure transmitters with overload protection, Automation and Remote Control 77(7): 1281-1285, http://dx.doi.org/10.1134/S0005117916070146.

Baptista, M.; Sankararaman, S.; de Medeiros, I.P.; Nascimento, C.; Prendinger, H.; \& Henriques, E.M.P. 2018. Forecasting fault events for predictive maintenance using data-driven techniques and ARMA modeling, Computers \& Industrial Engineering 115: 41-53, http://dx.doi.org/10.1016/j.cie.2017.10.033.

Bogdanov, S.P.; \& Basov, O.O. 2012. Prospects and problems of using wireless sensors with autonomous power supply, TUSUR Reports 2/1(26): 20-23.

Brüel; \& Kjær. 1991. Monitoring the state of machinery. DKBR 0660-11. Denmark: Nerum. 47 p.

Burduk, A.; \& Chlebus, E. 2009. Evaluation of the risk in production systems with a parallel reliability structure, EksploatacjaiNiezawodność- Maintenance and Reliability 2(42): 84-95.

Cook, D.; Das, S. 2005. Smart Environments. Technologies, protocols and applications. Hoboken NJ: Wiley-Interscience.

Crowder, M.; \& Lawless, J. 2007. On a scheme for predictive maintenance, European Journal of Operational Research 176: 1713-1722, http://dx.doi.org/10.1016/i.ejor.2005.10.051.

Curcuru, G.; Cocconcelli, M.; Rubini, R.; \& Galante, G.M. 2017. System monitoring and maintenance policies: A review, from https://iris.unimore.it/handle/11380/1144821, accessed March 20, 2018.

Freyden, J. 2005. Modern sensors. Reference book (Transl. from English by Yu.A. Zabalotnaya under the editorship of. E.L. Svintsova), Tekhnosfera, Moscow.

Gotra, Z.Yu.; \& Tchaikovsky, O.I. (Eds.). 1995. Sensors. Reference book, Kamenyar, Lviv.

Kozlova, E.I. 2009. Metrological support of information processing systems: A summary of lectures, BSU, Minsk.

Lu, C.J.; \& Meeker, W.Q. 1993. Using degradation measures to estimate time to failure distribution, Technometrics 35(2): 161-174, http://dx.doi.org/ 10.1080/00401706.1993.10485038.

Mobley, R.K. 2002. An introduction to predictive maintenance, Elsevier Science.

Prause, G.; Atari, S. 2017. On sustainable production networks for Industry 4.0, Entrepreneurship and Sustainability Issues 4(4): 421-431, http://dx.doi.org/10.9770/jesi.2017.4.4(2).

Rausand, M.; \& Hoyland, A. 2004. System reliability theory: Models, statistical methods and applications, Wiley. 
The International Journal

ENTREPRENEURSHIP AND SUSTAINABILITY ISSUES

ISSN 2345-0282 (online) http://jssidoi.org/jesi/

2018 Volume 6 Number 2 (December)

http://doi.org/10.9770/jesi.2018.6.2(2)

Rawi, Z. 2010. Machinery Predictive Analytics, In: SPE Intelligent Energy Conference and Exhibition, 23-25 March, Utrecht, the Netherlands, Society of Petroleum Engineers. http://dx.doi.org/10.2118/128559-MS.

Shakhnov, V.A.; Vlasov, A.I.; Rezchikova, E.V.; Tokarev, S.V.; Smurygin, I.M.; Denisenko, N.A.; \& Muravev, K.A. 2013. Method of functioning of the wireless sensor network. Patent for the invention of the Russian Federation No. 2556423.

Sharapov, V.M.; \& Polischuk, E.S. (Eds.). 2012. Sensors: Reference guide, Tekhnosfera, Moscow.

Stone, P. 2007. Introducing Predictive Analytics: Opportunities, http://dx.doi.org/10.2118/106865-MS.

Vlasov, A.I.; Yudin, A.V.; Salmina, M.A.; Shakhnov, V.A.; \& Usov, K.A. 2017a. Design methods of teaching the development of internet of things components with considering predictive maintenance on the basis of mechatronic devices, International Journal of Applied Engineering Research 12(20): 9390-9396.

Vlasov, A.I.; Grigoriev, P.V.; \& Zhalnin, V.P. 2017b. Application of the methods and means of radio frequency identification in the corporate information production systems, In: Proceedings of the International Symposium "Reliability and Quality" 1: $272-277$.

Vlasov, A.I.; Ivanov, V.V.; \& Kosolapov, I.A. 2011. Methods of proactive prediction of broadband network status, Software Products and Systems 1: 3-6.

Whitaker, D.A.; Egan, D.; O’Brien, E.; \& Kinnear, D. 2018. Application of multivariate data analysis to machine power measurements as a means of tool life predictive maintenance for reducing product waste, from https://arxiv.org/abs/1802.08338, accessed March 16, 2018.

Yudin, A.: Kolesnikov, M.; Vlasov, A.; \& Salmina, M. 2017. Project oriented approach in educational robotics: From robotic competition to practical appliance, Advances in Intelligent Systems and Computing 457: 83-94, http://dx.doi.org/10.1007/978-3-319-42975-5_8.

Andrey I. VLASOV, Cand.Sci. (Engineering), Associate professor of the department IU4 "Designing and Technology of Electronic Equipment", assistant manager as IU4 chair on scientific work of the Bauman Moscow State Technical University (Moscow, Russian Federation). Research interests: neural networks, stable operating systems, Internet of things (IoT), public-private partnerships, economic sustainability.

ORCID ID: orcid.org/0000-0001-5581-4982

Pavel V. GRIGORIEV, Master of the department IU4 "Designing and Technology of Electronic Equipment" of the Bauman Moscow State Technical University (Moscow, Russian Federation). Research interests: Big Data, wireless sensor networks, processing digital signal, Internet of things (IoT), stable operating systems.

ORCID ID: orcid.org/0000-0002-0716-3043

Aleksey I. KRIVOSHEIN, Postgraduate student of the department IU4 "Designing and Technology of Electronic Equipment" of the Bauman Moscow State Technical University (Moscow, Russian Federation). Research interests: technology predictive industrial maintenance, system analysis, modeling and optimization of processes.

ORCID ID: orcid.org/0000-0001-8292-452X

Vadim A. SHAKHNOV, Corresponding member of RAS, Dr.Sci. (Engineering), Professor, Head of the department IU4 "Designing and Technology of Electronic Equipment" of the Bauman Moscow State Technical University (Moscow, Russian Federation). Research interests: multidimensional data, industrial production, technological control, modeling and optimization of processes, knowledge management, professional education.

ORCID ID: orcid.org/0000-0003-4013-1905 
The International Journal

ENTREPRENEURSHIP AND SUSTAINABILITY ISSUES

ISSN 2345-0282 (online) http://jssidoi.org/jesi/

2018 Volume 6 Number 2 (December)

http://doi.org/10.9770/jesi.2018.6.2(2)

Sergey S. FILIN, Technical Director of the LLC "Connect" (Moscow, Russian Federation), Engineer of the department IU4 "Designing and Technology of Electronic Equipment" of the Bauman Moscow State Technical University (Moscow, Russian Federation). Research interests: industrial production, stable operating systems, Internet of things (IoT).

ORCID ID: orcid.org/0000-0002-6385-0454

Vladimir S. MIGALIN, Lead programmer of the LLC "Connect" (Moscow, Russian Federation). Research interests: stable operating systems, Internet of things (IoT), industrial production, intelligent analytical systems.

ORCID ID: orcid.org/0000-0002-9499-4469

Register for an ORCID ID:

https://orcid.org/register

Copyright (C) 2018 by author(s) and VsI Entrepreneurship and Sustainability Center

This work is licensed under the Creative Commons Attribution International License (CC BY).

http://creativecommons.org/licenses/by/4.0/

c) (i) Open Access 